\title{
Aggregation Number of Cholesterol Moieties in Hydrophobic Microdomains Formed by Self-Association of a Cholesterol-Bearing Polyelectrolyte in Water
}

\author{
Shin-ichi Yusa, ${ }^{\dagger}$ Kasumi IkedA, Tohei YAmamoto, and Yotaro MorishimA* \\ Department of Applied Chemistry, Himeji Institute of Technology, \\ 2167 Shosha, Himeji 671-2201, Japan \\ * Department of Macromolecular Science, Graduate School of Science, \\ Osaka University, Toyonaka, Osaka 560-0043, Japan
}

(Received August 15, 2002; Accepted September 17, 2002)

KEY WORDS Amphiphilic Polyelectrolyte / Cholesterol / Aggregation Number / Fluorescence /

Self-associating hydrophobically modified watersoluble polymers are of current scientific and technological interest because of their relevance to biological macromolecular systems and also because of a variety of practical applications. ${ }^{1}$ Hydrophobic interactions are a major driving force for the self-association of such polymers in water.

Previously, we reported the self-association of a random copolymer of sodium 2-(acrylamido)-2methylpropanesulfonate (NaAMPS) and cholesteryl 6methacryloyloxyhexanoate (Chol- $\left.\mathrm{C}_{5}-\mathrm{MA}\right)(5 \mathrm{~mol} \%)$ in water. ${ }^{2,3}$ The type of self-association of this copolymer was found to be rather unique in that the copolymer forms a "closed" type ${ }^{4}$ multipolymer micelle consisting of a number of hydrophobic domains (cores) in one polymer micelle and that the mean aggregation number $\left(N_{\mathrm{agg}}\right)$ of the cholesteryl groups in one hydrophobic microdomain is very small (i.e., $N_{\text {agg }}=17-$ 19). These findings with the NaAMPS/Chol- $\mathrm{C}_{5}-\mathrm{MA}$ copolymer are strikingly different from those with a random copolymer of NaAMPS and dodecyl methacrylate (DMA) (9 mol\%). ${ }^{5}$ Namely, the NaAMPS/DMA copolymer forms a multipolymer micelle consisting of a single hydrophobic microdomain with $N_{\text {agg }}$ of dodecyl groups of $c a$. 195 . These two NaAMPS copolymers are similar in structure except a cholesterol moiety is attached to each hydrophobic monomer unit via a $\mathrm{C}_{5}$ aliphatic chain in NaAMPS/Chol- $\mathrm{C}_{5}-\mathrm{MA}$ whereas a $\mathrm{C}_{12}$ aliphatic chain is attached to each hydrophobic monomer unit in NaAMPS/DMA. The small $N_{\text {agg }}$ observed for NaAMPS/Chol- $\mathrm{C}_{5}-\mathrm{MA}$ may be attributed in part to the intrinsic nature of cholesterol moieties comprised of rigid steroid rings and in part to steric constraints exerted to the rigid hydrophobes by polymer

${ }^{\dagger}$ To whom correspondence should be addressed. main chain.

To examine the effect of steric constraints by polymer backbone on the aggregation number of cholesterol moieties, we synthesized cholesterol-bearing NaAMPS copolymers where cholesteryl groups are less restricted by polymer chain, i.e., cholesteryl groups are linked to the polymer backbone via a longer alkyl chain (i.e., $\mathrm{C}_{10}$ chain). In these copolymers, cholesteryl groups are expected to undergo self-association under less constraining conditions than in the case of NaAMPS/Chol- $\mathrm{C}_{5-}$ MA.

In this paper, we have reported the synthesis of the copolymers of NaAMPS and cholesteryl 11methacrylamidoundecanoate $\left(\mathrm{Chol}-\mathrm{C}_{10}-\mathrm{MAm}\right)$ with varying compositions (Figure 1) and their self-associa-

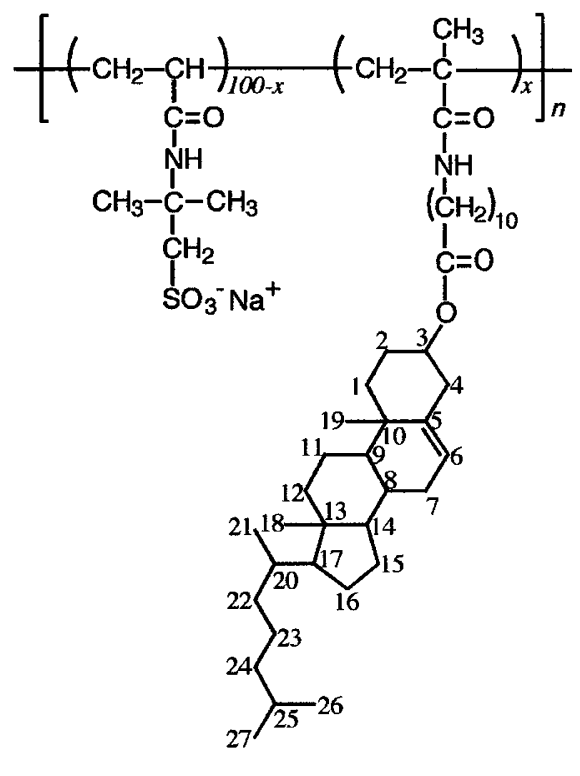

$$
x\left(f_{\text {Chol }}\right)=0,1,5,10,20 \text {, and } 30 \mathrm{~mol} \%
$$

Figure 1. Structures of NaAMPS/Chol- $\mathrm{C}_{10}$-MAm copolymers. 
tion in water focusing on $N_{\text {agg }}$ of cholesteryl groups.

\section{EXPERIMENTAL}

Chol- $\mathrm{C}_{10}-\mathrm{MAm}$ was synthesized according to the method reported by Shibaev. ${ }^{6}$ Pyrene, and 3,4dimethylbenzophenone (DMBP) were recrystalized from methanol. Copolymers of NaAMPS and Chol$\mathrm{C}_{10}$-MAm were prepared by ordinary free-radical copolymerization in homogeneous solution using $N, N$ dimethylformamide as a solvent. ${ }^{7}$ The copolymer compositions, determined by ${ }^{1} \mathrm{H}$ NMR spectroscopy, were the same as those in the monomer feed. From GPC with use of a mixture of DMSO/DMF $(60 / 40$, v/v) containing $50 \mathrm{mM}$ of $\mathrm{LiBr}$ as eluent, weight-average molecular weight $\left(M_{\mathrm{w}}\right)$ of the copolymers and NaAMPS homopolymer (polyNaAMPS) were roughly estimated as listed in Table I.

Fluorescence spectra were recorded on a Shimadzu RF-5000 fluorescence spectrophotometer. Emission spectra of pyrene were measured with excitation at $334 \mathrm{~nm}$. Excitation spectra of pyrene were monitored at $390 \mathrm{~nm}$. Sample solutions were prepared by dissolving a known amount of polymer in pyrene containing water $\left(5 \times 10^{-7} \mathrm{M}\right)$, and the solutions were allowed to stand for a day. Pyrene containing water was prepared as reported previously. ${ }^{2}$

Time-resolved fluorescence quenching (TRFQ) experiments were carried out by a single photon counting technique using a Horiba NAES-550 system. Sample solutions were prepared as follows. Predetermined amounts of pyrene and DMBP were dissolved together in aqueous solution containing the copolymers and the sample solution was deaerated by purging with Ar gas for $30 \mathrm{~min}$ prior to measurement. Pyrene was excited at $334 \mathrm{~nm}$ and its fluorescence was monitored at $400 \mathrm{~nm}$. In the absence of DMBP, pyrene fluorescence decays are single-exponential with a decay rate constant $k=$ $1 / \tau_{0}$, where $\tau_{0}$ is the fluorescence lifetime of pyrene solubilized in cholesteryl aggregates of the copolymer. Fluorescence decay data in the presence of DMBP was fitted to the Infelta-Tachiya equation derived for fluorescence quenching in micelles assuming the distribution of fluorescence probe molecules over the micelle is frozen on the time scale of the fluorescence lifetime ${ }^{8,9}$

$$
I(t)=I(0) \exp \left[-\frac{t}{\tau_{0}}-R\left\{1-\exp \left(-k_{\mathrm{Q}} t\right)\right\}\right]
$$

where $I(t)$ and $I(0)$ are the fluorescence intensities at time $t$ and zero, respectively, following the excitation, $R$ is the average number of quenchers in the cholesterol microdomain, and $k_{\mathrm{Q}}$ is the first-order quenching rate constant. $N_{\text {agg }}$ is obtained from
Table I. Characteristics of the polymers

\begin{tabular}{cccc}
\hline$f_{\text {Chol }}(\mathrm{mol} \%)$ & $M_{\mathrm{w}} \times 10^{5 \mathrm{a}}$ & $N_{\text {Chol }}{ }^{\mathrm{b}}$ & $N_{\mathrm{agg}}{ }^{\mathrm{c}}$ \\
\hline 0 & 1.1 & & \\
1 & 1.2 & 1 & 17 \\
5 & 1.4 & 5 & 12 \\
10 & 1.5 & 10 & 14 \\
\hline
\end{tabular}

${ }^{\mathrm{a}}$ Determined by GPC using a $50-\mathrm{mM} \mathrm{LiBr}$ DMSO/DMF (60/40, v/v) solution as an eluent. ${ }^{b}$ Number of cholesteryl groups per one polymer chain. ${ }^{\mathrm{c}}$ Average aggregation number of cholesteryl groups in one hydrophobic microdomain.

$$
N_{\text {agg }}=R \frac{[\mathrm{Chol}]}{[\mathrm{Q}]}
$$

where [Chol] and [Q] are the concentrations of the cholesteryl group and quencher, respectively.

\section{RESULTS AND DISCUSSION}

The copolymers of NaAMPS and Chol- $\mathrm{C}_{10}-\mathrm{MAm}$ with Chol- $\mathrm{C}_{10}-\mathrm{MAm}$ contents $\left(f_{\text {Chol }}\right)$ of $0,1,5,10$, 20 , and $30 \mathrm{~mol} \%$ were prepared. The copolymers were soluble in water when $f_{\text {Chol }} \leq 10 \mathrm{~mol} \%$ but they were insoluble when $f_{\text {Chol }}=20$ and $30 \mathrm{~mol} \%$. Therefore, we employed only the copolymers with $f_{\text {Chol }} \leq 10 \mathrm{~mol} \%$ for studies of their self-association in water. Since hydrophobes are connected to the main chain via amide bonds, the NaAMPS/Chol- $\mathrm{C}_{10}-\mathrm{MAm}$ copolymers are more soluble in water ${ }^{5}$ than the NaAMPS/Chol- $\mathrm{C}_{5^{-}}$ MA copolymers in the previous work, the latter being soluble in water only when $f_{\text {Chol }} \leq 5 \mathrm{~mol} \%$. Average numbers of the Chol- $\mathrm{C}_{10}-\mathrm{MAm}$ units per one polymer chain $\left(N_{\text {Chol }}\right)$ were roughly calculated from the number-average molecular weights estimated from GPC and Chol- $\mathrm{C}_{10}-\mathrm{MAm}$ contents for the copolymers with $f_{\text {Chol }}=1,5$, and $10 \mathrm{~mol} \%$ (Table I).

Figure 2 a shows the intensity ratio of the third to first vibronic peaks $\left(I_{3} / I_{1}\right)$ for pyrene in the presence of polyNaAMPS and the copolymers with $f_{\text {Chol }}=1,5$, and $10 \mathrm{~mol} \%$ as a function of polymer concentrations $\left(C_{\mathrm{p}}\right)$. It has been established that $I_{3} / I_{1}$ decreases with increasing the micropolarity. ${ }^{10-12}$ For low $C_{\mathrm{p}}, I_{3} / I_{1}$ for all the polymers are estimated to be $0.57-0.60$, similar values as that in water. $I_{3} / I_{1}$ in the presence of polyNaAMPS is almost constant at $0.57-0.63$, independent of $C_{\mathrm{p}}$ over the whole range examined. In the case of the NaAMPS/Chol- $\mathrm{C}_{10}-\mathrm{MAm}$ copolymers, $I_{3} / I_{1}$ commences to increase significantly at a certain $C_{\mathrm{p}}$ as $C_{\mathrm{p}}$ is increased. The polymer concentration for the commencement of the increase in $I_{3} / I_{1}$ upon an increase in $C_{\mathrm{p}}$ depends strongly on $f_{\text {Chol }}$, and this commencement of $C_{\mathrm{p}}$ is much lower for higher $f_{\text {Chol }}$. For the copolymer with $f_{\text {Chol }}=1 \mathrm{~mol} \%$, the commencement of $C_{\mathrm{p}}$ is 


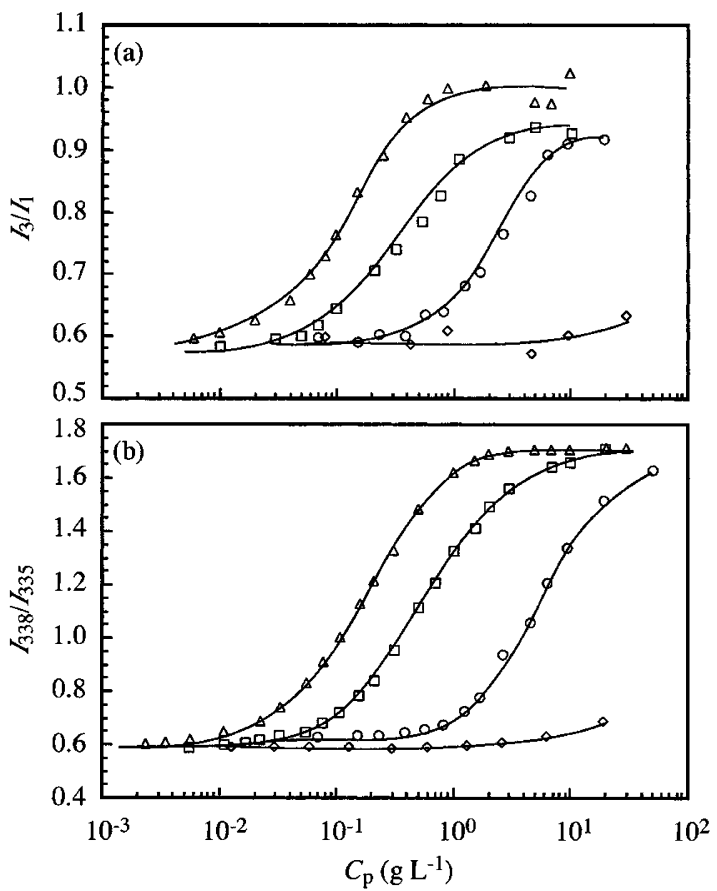

Figure 2. Plots of $I_{3} / I_{1}$ (a) and $I_{338} / I_{335}$ (b) for pyrene $\left(5 \times 10^{-7}\right.$ $\mathrm{M})$ as a function of $C_{\mathrm{p}}$ for the copolymers with $f_{\text {Chol }}=1(\bigcirc), 5(\square)$, and $10 \mathrm{~mol} \%(\triangle)$. Plots for polyNaAMPS $(\diamond)$ are presented for comparison.

more than one order of magnitude higher than that for the copolymer with $f_{\text {Chol }}=10 \mathrm{~mol} \%$.

The 0-0 band for pyrene excitation spectra in water shows a peak at $335 \mathrm{~nm}$ whereas it is observed at $338 \mathrm{~nm}$ when pyrene is solubilized in hydrophobic microdomains. ${ }^{73-15}$ Therefore, it is established that a critical aggregation concentration for amphiphilic molecules can be determined by $I_{338} / I_{335}$ for the $0-0$ band in pyrene excitation spectra, where $I_{335}$ and $I_{338}$ are the pyrene emission intensity at 335 and $338 \mathrm{~nm}$, respectively. In Figure $2 \mathrm{~b}, I_{338} / I_{335}$ is plotted as a function of $C_{\mathrm{p}} \cdot I_{338} / I_{335}$ for polyNaAMPS shows a value of pyrene in water over the whole range of $C_{\mathrm{p}}$ examined. $I_{338} / I_{335}$ for each polymer exhibits a significant increase at a certain $C_{\mathrm{p}}$ that is close to $C_{\mathrm{p}}$ for the onset of the increase in $I_{3} / I_{1}$. These observations indicated that the cholesteryl groups formed a hydrophobic microdomain where pyrene is solubilized.

Figure 3 shows decay profiles for pyrene fluorescence with and without DMBP in the presence of the copolymer with $f_{\text {Chol }}=5 \mathrm{~mol} \%$ in water at $C_{\mathrm{p}}=$ $40 \mathrm{~g} \mathrm{~L}^{-1}$. The decay data in the absence of DMBP were reasonably well-fitted to a single-exponential function. On the other hand, the decays in the presence of the quencher were well-fitted to eq 1 . A value of $\tau_{0}$ for pyrene solubilized in cholesterol microdomains was estimated using a conventional deconvolution method to be $312 \mathrm{~ns}$. Therefore, in the fitting procedure for fluorescence decay data in the presence of DMBP, $\tau_{0}$ in

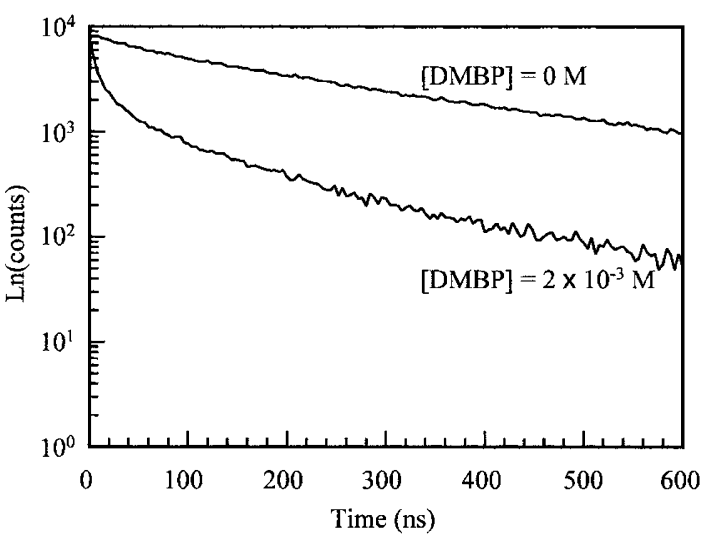

Figure 3. Fluorescence decays for pyrene $\left(5 \times 10^{-7} \mathrm{M}\right)$ solubilized in aqueous solutions of the copolymer with $f_{\text {Chol }}=5 \mathrm{~mol} \%$ at $C_{\mathrm{p}}=40 \mathrm{~g} \mathrm{~L}^{-1}$ in the absence and presence of DMBP $\left(2 \times 10^{-3} \mathrm{M}\right)$.

eq 1 was constrained to $312 \mathrm{~ns}$. The $N_{\text {agg }}$ value calculated from eq 2 for the copolymer with $f_{\text {Chol }}=5 \mathrm{~mol} \%$ at $C_{\mathrm{p}}=40 \mathrm{~g} \mathrm{~L}^{-1}$ was 12 . In the case of the copolymers with $f_{\text {Chol }}=1$ and $10 \mathrm{~mol} \%, N_{\text {agg }}$ values were estimated to be 17 and 14, respectively. As described earlier, the $N_{\text {Chol }}$ values are 1,5 , and 10 for the copolymers with $f_{\text {Chol }}=1,5$, and $10 \mathrm{~mol} \%$, respectively (Table I). Assuming all pendent cholesteryl groups participate in the event of their aggregation, the copolymer with $f_{\text {Chol }}=1 \mathrm{~mol} \%$, for example, may form a unicore micelle composed of about 17 polymer chains.

The values of $N_{\text {agg }}$ for the copolymers with $f_{\text {Chol }}=$ $1-10 \mathrm{~mol} \%$ were found to be in the range $12-17$. These values are similar to those observed for NaAMPS/Chol$\mathrm{C}_{5}-\mathrm{MA}(5 \mathrm{~mol} \%)$ copolymer $\left(N_{\mathrm{agg}}=17-19\right)$. Thus, we can conclude that cholesterol moieties in the NaAMPS copolymers form hydrophobic microdomains with small aggregation numbers independent of the spacer length and copolymer composition (i.e., $N_{\text {chol }}$ ). The conclusion from this study suggests that the similarly small $N_{\text {agg }}$ values observed for the cholesterol-bearing NaAMPS copolymers are attributed to the inherent nature of cholesterol moieties.

Akiyoshi and co-workers ${ }^{16-19}$ have reported that pullulan covalently modified with a few cholesteryl groups (CHP) forms stable nanoparticles consisting of cholesterol aggregates with $N_{\text {agg }}$ ranging from 3.5 to 5.7. These values are essentially the same independent of the molecular weight of the parent pullulan and the degree of substitution with the cholesteryl group.

Cholesterols and bile salts resemble each other in rigidity and chemical structure of steroid rings. Bile salts, such as sodium cholate and sodium deoxycholate possessing hydrophobic and dydrophilic groups, form micelle-like aggregates in water. For example, sodium cholate forms aggregates with $N_{\text {agg }}$ ranging from 12 to 19. ${ }^{20,21}$ It has been also reported that the aggregate of 
sodium deoxycholate bearing a sulfonate ion in water contains about 11 bile salt molecules. ${ }^{22}$

The small aggregation numbers of cholesterol groups observed in the present study are consistent with these reported values of the aggregation numbers for cholesterols and bile salts, arising from the inherent nature of steroid compounds with rigid ring structures.

\section{REFERENCES}

1. C. L. McCormick, J. Bock, and D. N. Schultz, in "Encyclopedia of Polymer Science and Engineering”, John Wiley \& Sons, Inc., New York, N.Y., 1989, vol. 17.

2. S. Yusa, M. Kamachi, and Y. Morishima, Langmuir, 14, 6059 (1998).

3. S. Yusa, A. Hashidzume, and Y. Morishima, Langmuir, 15, 8826 (1999).

4. H. G. Elias, J. Macromol. Sci., Part A, 7, 601 (1973).

5. T. Noda and Y. Morishima, Macromolecules, 32, 4631 (1999).

6. V. P. Shibaev, N. A. Pleté, and Y. S. Freidzon, J. Polym. Sci., Polym. Chem. Ed., 17, 1655 (1979).

7. T. Noda, A. Hashidzume, and Y. Morishima, Macromolecules, 33, 3694 (2000).

8. P. P. Infelta, M. Grätzel, and J. K. Thomas, J. Phys. Chem., 78, 190 (1974).
9. M. Tachiya, Chem. Phys. Lett., 33, 289 (1975).

10. K. Kalyanasundaram and J. K. Thomas, J. Am. Chem. Soc., 99, 2039 (1977).

11. H. Faes, F. C. D. Schryver, K. Bijman, J. Kevelam, and J. B. F. N. Engbert, Macromolecules, 29, 3875 (1996).

12. K. Procházka, T. J. Martin, P. Munk, and S. E. Webber, Macromolecules, 29, 6518 (1996).

13. M. Wilhelm, C. Zhao, Y. Wang, R. Xu, M. A. Winnik, J. Mura, G. Riess, and M. D. Croucher, Macromolecules, 24, 1033 (1991).

14. I. Astafieva, X. F. Zhong, and A. Eisenberg, Macromolecules, 26, 7339 (1993).

15. C. Kim, S. C. Lee, S. W. Kang, I. C. Kwon, Y. H. Kim, and S. Y. Jeong, Langmuir, 16, 4792 (2000).

16. K. Akiyoshi, K. Nagai, T. Nishikawa, and J. Sunamoto, Chem. Lett., 1727 (1992).

17. K. Akiyoshi, S. Deguchi, N. Moriguchi, S. Yamaguchi, and J. Sunamoto, Macromolecules, 26, 3062 (1993).

18. S. Deguchi, K. Akiyoshi, and J. Sunamoto, Macromol. Rapid Commun., 15, 705 (1994).

19. T. Nishikawa, K. Akiyoshi, and J. Sunamoto, Macromolecules, 27, 7654 (1994).

20. G. Benzonana, Biochim. Biophys. Acta., 176, 836 (1969).

21. K. S. Birdi, Finn. Chem. Lett., 142 (1982).

22. R. Zana and D. Guveli, J. Phys. Chem., 89, 1687 (1985). 